\title{
Positive Youth Development Across Cultures: Introduction to the Special Issue
}

\author{
Nora Wiium ${ }^{1}$ (D) $\cdot$ Radosveta Dimitrova ${ }^{1,2}$ \\ Published online: 12 January 2019 \\ (c) Springer Science+Business Media, LLC, part of Springer Nature 2019
}

\begin{abstract}
Background Promoting well-being of young people within the positive youth development (PYD) framework has been found to be promising. The basic models of PYD-the 5C's (competence, confidence, character, connection, and caring) and developmental assets (significant relationships, skills, opportunities and values that promote thriving exemplified by external assets or environmental resources and internal assets or interpersonal strengths), have been largely shown to promote positive aspects of development among diverse samples of young people. However, PYD research has mainly been conducted within the US context.
\end{abstract}

Objectives Explore the generalizability of the PYD framework to understand and promote positive aspects of development in young people beyond the US context.

Method Six papers using cross-sectional methods reported data on adolescents and/or emerging adults $(N=6820)$ with diverse cultural backgrounds from ten countries (Brazil, China, El Salvador, Ghana, Italy, Kenya, Norway, Slovenia, South-Africa, Turkey) in four continents (Africa, Asia, Europe and Latin America). Widely applied PYD scales developed within the US and adequately adapted in each cultural context were used for data collection.

Results The generalizability of the PYD framework (the presence and positive relations between the 5Cs and developmental assets in promoting optimal development) was largely confirmed among culturally diverse samples of young people investigated in the papers.

Conclusion While the findings of the current special issue extend the generalizability of the PYD framework beyond the US context, more research is needed to ascertain appropriate developmental assets to facilitate PYD, as defined by the specific context where young people are embedded.

Keywords PYD · 5Cs · Developmental assets · Cross-national project · Adolescents · Emerging adults

Nora Wiium

Nora.Wiium@uib.no

1 Faculty of Psychology, University of Bergen, Christiesgate 12, 5020 Bergen, Norway

2 Department of Psychology, Stockholm University, Stockholm, Sweden 


\section{Introduction}

At the turn of the twentieth century, a new line of research that focuses on the strengths of young people rather than on their weaknesses emerged (see Benson et al. 2006; Damon 2004; Lerner et al. 2015). In contrast to the deficit approach, which has dominated adolescent research since Stanley Hall's (1904) publication on adolescence as a period of storm and stress, the PYD perspective highlights youth strengths and depicts youth as potential human resources for societal development. PYD argues that an alignment of youth personal skills and competencies with the positive developmental experiences of relationship and opportunities in their contexts will facilitate the development of self and society (Lerner et al. 2015). This assertion has been studied mainly in US samples although recent publications have seen growing interest of the PYD perspective in non-US research (e.g., Leman et al. 2017). The six articles in the present special issue extend PYD research beyond the US context and adopt such perspective in a global international context.

Besides the contribution by Tirrell et al. (2018), all papers included in the special issue are based on the Positive Youth Development Cross-National project that was initiated at the University of Bergen, Norway, in 2014. The project's novelty lies in working with culturally diverse youth within and between countries. In addition, the project adopts a comprehensive theoretical approach of Bronfenbrenner's ecological systems theory (Bronfenbrenner and Morris 1998), Benson's developmental assets (Benson 2007) and Lerner's 5Cs of PYD (Lerner et al. 2015) to examine youth's developmental outcomes within a bidirectional interaction with their immediate family, peers, school and community. PYD aims at equipping youth for their present and future tasks and has typically been indexed by the 5Cs of Competence (academic, social, vocational skills), Confidence (sense of mastery, positive identity, self-worth), Character (integrity, moral commitment, personal values), Connection (healthy relation to community, friends, family, school) and Caring (empathy and sympathy).

Currently, the project brings together partners from over 20 countries located in Africa, Asia, Australia, Europe, Middle East, Latin America and the United States, representing expertise in diverse disciplines of psychology, public health, environmental science, sociology, family studies and implementation science. The ongoing data collection involves more than 8000 minority and majority youth (ages 16-29) from participating countries selected based on theoretical considerations (sufficient cultural variability and level of economic development) and logistical reasons (developed networks and previously successfully collected data). The overall aim of the cross-national project is to advance knowledge about PYD mechanisms in culturally diverse settings and develop a basis for better policy measures in promoting youth well-being, under the PYD framework.

\section{Overview of this Special Issue}

The aim of this special issue is to generate new insight on PYD and positive adaptation of youth living in underrepresented cultures, as well to highlight innovative research examples. The contributions presented here promote novel research ideas on positive adaptation of youth living in contexts where very little or no research on PYD has been done and psychological phenomena or strengths for young people may greatly differ from those proposed by well-known US-based PYD work. In so doing, we present new data on culturally 
diverse samples from various continents or regions (Africa, Asia, Europe and Latin America) and highlight cultural variations in optimal developmental adaptations among youth.

In the first paper, Chen et al. (2017) examined the relationships between environmental certainty (i.e., judging the chances of attaining different outcomes in an environment of resource acquisition, social rank, or offspring survivability in the future), life history strategies (LHS) and developmental assets (external and internal) among adolescents. Crosssectional data was collected from 577 students attending one of five public high schools in Shanghai, People's Republic of China. Positive correlations between developmental assets and a slow LHS, where participants who reported more assets were also likely to report more favorable environmental conditions were observed. In addition, an indirect effect of environmental certainty on developmental assets through a slow LHS was found; meaning that participants who perceived that their environmental conditions were predictable were also more likely to report the conditions as favorable, and in turn more likely to report both external and internal developmental assets. Chen and colleagues' contribution represents one of the first studies to examine PYD within an evolutionary life history framework.

The second contribution led by Luciana Dutra-Thomé examined the associations of risk (exposure to domestic and community violence; prejudice) and promotive factors (family, college, and community connectedness) with self-concept factors (self-esteem and selfefficacy) and problem behaviors (licit drug use, sex risk behavior, and antisocial behavior) among 547 emerging adults in a Brazilian urban context. The results indicated significant associations between high levels of family connectedness and high self-esteem, in addition to a significant association between higher levels of college connectedness (connection with people at the educational institutions) and high levels of self-esteem and self-efficacy. Besides highlighting the importance of individuals' relationships with people within their family and educational contexts on their self-concept, Dutra-Thomé et al. (2018) paper takes a novelty approach by investigating contextual and individual variables in an environment characterized by socioeconomic inequality, insecurity, and violence.

In the third contribution, Wiium et al. (2018) extend the scope of PYD research by assessing internal (individual qualities, values, skills, and self-perceptions: commitment to learning, positive values, social competencies and positive identity) and external assets (experiences and relationships across multiple contexts: support, empowerment, boundaries and expectations and constructive use of time) among secondary school students in Italy $(n=526)$, Norway $(n=592)$ and Turkey $(n=116)$. In addition, interactions with gender and parents' educational level were examined. For internal assets, youth in Norway and Turkey reported the highest number of commitment to learning assets; youth in Norwaythe highest number of assets on social competence, youth in Turkey - the highest number of assets on positive identity and girls in Norway-the most internal assets. For external assets, youth in Norway and Turkey reported more support assets than those in Italy; youth in Norway reported the highest number of assets on empowerment, as well as boundaries and expectations, while their counterparts in Italy and Turkey reported more assets on constructive use of time. Girls in Norway reported more assets on support, empowerment, boundaries and expectations. An interaction term between country and father's educational level revealed that youth in Italy whose father had higher education reported the highest number of constructive use of time assets. Wiium and colleagues' article illustrates how national, parents and individuals' characteristics can influence the experience of developmental assets.

The fourth contribution by Adams et al. (2018) examined the importance of developmental assets for academic performance in three sub-Saharan African contexts: Ghana, Kenya, and South Africa. In a cross-sectional study, secondary school students in Ghana 
$(n=428)$, Kenya $(n=180)$, and South Africa $(n=184)$ were asked to complete a survey containing an adapted version of the Benson's (2007) 40 assets as well as provide information on their school grades. Results showed that Ghanaian students reported fewer assets compared to Kenyan and South African students, and across all countries, adolescents with good academic performance reported more developmental assets. Adams et al. (2018) observed that students who drew on their internal assets relative to those who did not, were more likely to perform better in school, notwithstanding their country of origin.

In the fifth contribution by Kozina et al. (2018), the authors assessed the associations between connection, caring, confidence and character and academic (math) achievement among youth in Slovenia, a context that reflects a homogeneous population and contemporary transition to democracy. Variability across gender and school types (i.e., general gymnasium, technical gymnasium, Technical education programs, Vocational programs of medium duration, and Vocational programs of short duration) was also examined. Data was collected from a large sample of 15 -year-olds $(N=2802)$ who had recently transitioned to upper-secondary. Positive association between math achievement and student's self-reported confidence and a negative one between character and connection emerged. Concerning variability by gender and school types, confidence was positively associated with math achievement in general gymnasiums, in technical programs and among boys; caring was negatively associated with math achievement in technical gymnasiums; connection was negatively associated with math achievement in technical programs; character was positively associated with math achievement in vocational programs of medium duration.

The sixth and final contribution by Tirrell et al. (2018) applied PYD measures to compare youth enrolled in compassion international (CI) programs, a faith-based childsponsorship organization committed to alleviating child poverty and promoting thriving, to youth not enrolled in these programs. Survey data was collected from 888 youth, ages 9-15 years, living in El Salvador, Latin America, half of whom were enrolled in CI programs. CI-supported youth were found to report higher levels of Transcendence (spirituality) and Character (one of the 5Cs of PYD) than non-CI-supported youth. In addition, CI-supported youth demonstrated a significant relation between Character and Connection contrary to non-CI-supported youth. Tirrell and colleagues' paper represents the inaugural step in answering questions regarding the integrated and comprehensive measurement of thriving for global youth living in poverty.

\section{Incremental Value to the PYD Field and Prospects}

The rise in scope and significance of PYD has led to an increased attention and related work in the fields of developmental, educational, and acculturation psychology. Such considerable expansion has witnessed similar special sections adopting a PYD framework in Child Development focusing on PYD in diverse and global contexts (Leman et al. 2017), Child Development's Perspectives on minority children (Cabrera et al. 2012), and Journal of Adolescence's Positive adaptation of immigrant and minority youth (Titzmann et al. 2018). These scholarly contributions inform PYD research, intervention, global and local policy by enhancing optimal well-being, thriving and success among marginalized and culturally diverse youth. Yet, these scholarly contributions primarily focus on immigrant and ethnic minority children and youth mainly US-based and limited geographical regions.

This issue builds on such scholarly work by providing unique incremental contributions to the PYD field in several highly original and innovative ways. First, insofar 
as little PYD work has been devoted to culturally diverse populations of youth across countries, such as Brazil, China, El Salvador, Ghana, Italy, Kenya, Norway, Slovenia, South-Africa, Turkey and continents (Africa, Asia, Europe, and Latin America). Thus, this special issue increases knowledge about young populations who are often neglected or disparaged, with little attention directed toward the understanding of their strengths and interactions with families and communities. Second, all included contributions address the pressing need to develop and adapt adequate measures in culturally diverse samples as to optimize and broaden PYD research in a global context. This is of utmost importance as we provide sound measurement tools to study PYD models in new or previously understudied samples and broader areas of developmental growth. Third, we add new knowledge to the field by extending PYD models to emerging adults who are of particular relevance since young people in the 18-29 age group are being characterized by identity exploration, instability, longer education, later entry into stable work, later marriage, and later parenthood (Arnett 2018). The features of this developmental stage are closely related to PYD and ways to promote optimal well-being and thriving as well as optimal transition to adulthood. Fourth, the inclusion of PYD studies with their individual or comparative focus on countries provides a unique analysis of young populations in a wide range of contexts as well as drawing inferences about commonalities and differences in their experience across cultures and nations. Therefore, this special issue adds an enormous contribution to the next generation of studies and work in the PYD field by broadening our knowledge on how to promote and facilitate the emergence of a range of desirable attributes, skills and outcomes among young people in various countries and regions.

Finally, the special issue is complemented by two commentaries authored by leading scholars in developmental and intervention science. We were particularly interested in providing experts' opinions as to identify crucial emerging themes, and present the challenges to be addressed in the PYD field in a global and multidisciplinary prospective in the years ahead. Lerner and Chase's (2018) commentary highlights among others the importance of sound developmental theory and conceptualization, as well as innovations in methodology in describing, explaining and optimizing thriving among the diverse youth of the majority world. Eichas et al. (2019) deliberate on how connecting PYD to the science and practice of treatment and prevention will ensure achieving a more fully integrated intervention science. Together, the two commentaries outline how PYD research and intervention can be advanced to facilitate optimal development in youth embedded in different cultures and countries.

In conclusion, the articles in this special issue discuss the roles of selected, central promotive factors in the genesis and development of PYD across cultures. Taken together, they indicate that several central psychological factors (e.g., promotive contexts, positive experiences, relationships, and family and community strength) contribute to the emergence of PYD. These factors along with broader environmental triggers are central to our understanding of PYD and importantly, its intervention in understudied cultures. This special issue provides unique selection of freshly produced work that brings together novel approaches to the study of PYD across culturally diverse populations and provides a timely relevant overview of contemporary research in PYD and its applications.

Acknowledgements This research was funded by the Faculty of Psychology and the Strategic Programme for International Research and Education (SPIRE) grant, both at the University of Bergen, Norway. We would like to extend our appreciation to all authors who contributed to the articles and commentaries in this issue. 


\section{Compliance with Ethical Standards}

Conflict of interest The authors declare that they have no conflict of interest.

Ethical Approval This article does not contain any studies with human participants performed by any of the authors.

\section{References}

Adams, B. G., Wiium, N., \& Abubakar, A. (2018). Developmental assets and academic performance of adolescents in Ghana, Kenya, and South Africa. Child \& Youth Care Forum. https://doi. org/10.1007/s10566-018-9480-z.

Arnett, J. J. (2018). Foreword. In R. Dimitrova (Ed.), Well-being of youth and emerging adults across cultures. New York: Springer.

Benson, P. L. (2007). Developmental assets: An overview of theory, research, and practice. In R. Silbereisen \& R. Lerner (Eds.), Approaches to positive youth development (pp. 33-59). London: SAGE Publications Ltd.

Benson, P. L., Scales, P. C., Hamilton, S. F., \& Semsa, A., Jr. (2006). Positive youth development: Theory, research, and applications. In W. Damon \& R. M. Lerner (Eds.), Theoretical models of human development. Volume 1 of Handbook of child psychology (6th ed.). Hoboken, NJ: Wiley.

Bronfenbrenner, U., \& Morris, P. (1998). The ecology of developmental processes. In W. Damon (Series Ed.), \& R. Lerner (Vol. Ed.), Handbook of child psychology: Vol. 1. Theoretical models of human development (5th ed., pp. 993-1028). New York: Wiley.

Cabrera, N. J., Beeghly, M., \& Eisenberg, N. (2012). Positive development of minority children: Introduction to the special issue. Child Development Perspectives, 6, 207-209. https://doi.org/10.111 1/j.1750-8606.2012.00253.x.

Chen, B.-B., Wiium, N., \& Dimitrova, R. (2017). A life history approach to understanding developmental assets among Chinese adolescents. Child \& Youth Care Forum. https://doi.org/10.1007/s1056 6-017-9433-y.

Damon, W. (2004). What is positive youth development? The Annals of the American Academy of Political and Social Science, 591, 13-24.

Dutra-Thomé, L., DeSousa, D., \& Koller, S. H. (2018). Promotive and risk factors for positive youth development among emerging adults in Brazil. Child \& Youth Care Forum. https://doi.org/10.1007/ s10566-018-9475-9.

Eichas, K., Ferrer-Wreder, L., \& Olsson, T. M. (2019). Contributions of positive youth development to intervention science. Child \& Youth Care Forum. https://doi.org/10.1007/s10566-018-09486-1.

Hall, G. S. (1904). Adolescence: Its psychology and its relation to physiology, anthropology, sociology, sex, crime, religion, and education (Vols. $1 \& 2$ ). Englewood Cliffs, NJ: Prentice Hall.

Kozina, A., Wiium, N., Gonzalez, J.-M., \& Dimitrova, R. (2018). Positive youth development and academic achievement in Slovenia. Child \& Youth Care Forum. https://doi.org/10.1007/s1056 6-018-9457-y.

Leman, P. J., Smith, E. P., Petersen, A. C., \& SRCD Ethnic-Racial Issues and International Committees. (2017). Introduction to the special section of Child development on positive youth development in diverse and global contexts. Child Development, 88, 1039-1044.

Lerner, R. M., \& Chase, P. A. (2018). Enhancing theory and methodology in the international study of positive youth development: A commentary. Child \& Youth Care Forum. https://doi.org/10.1007/ s10566-018-9485-7.

Lerner, R. M., Lerner, J. V., Bowers, E., \& Geldhof, G. J. (2015). Positive youth development: A relational developmental systems model. In W. F. Overton \& P. C. Molenaar (Eds.), Handbook of child psychology and developmental science. Vol. 1: Theory and method (7th ed., pp. 607-651). Hoboken, NJ: Wiley.

Tirrell, J. M., Geldhof, G. J., King, P. E., Dowling, E. M., Sim, A., Williams, K., et al. (2018). Measuring spirituality, hope, and thriving among Salvadoran youth: Initial findings from the Compassion International Study of Positive Youth Development. Child \& Youth Care Forum. https://doi. org/10.1007/s 10566-018-9454-1. 
Titzmann, P., Ferrer-Wreder, L., \& Dimitrova, R. (2018). Introduction to a special section on explaining positive adaptation of immigrant and minority youth across cultures. Journal of Adolescence, 62, 171-174. https://doi.org/10.1016/j.adolescence.2017.09.008.

Wiium, N., Dost-Gözkan, A., \& Kosic, M. (2018). Developmental assets among young people in three European contexts: Italy, Norway and Turkey. Child \& Youth Care Forum. https://doi.org/10.1007/ s10566-018-9446-1.

Publisher's Note Springer Nature remains neutral with regard to jurisdictional claims in published maps and institutional affiliations. 